\title{
Frecuencia de Virus Papiloma Humano en Tumores no Ginecológicos de la Región de la Araucanía, Chile
}

\author{
Human Papilloma Virus Frequency in non-Gynecological \\ Tumors in the Araucanía Region, Chile

\begin{abstract}
René Hoffstetter*; Alejandra Andana*; Pablo Guzmán*; Carmen G. Ili*;
\end{abstract} \\ Javier Retamal"; Bárbara Mora*; Juan C. Roa*** \& Raúl Sánchez ${ }^{* *}$
}

HOFFSTETTER, R.; ANDANA, A.; GUZMÁN, P.; ILI, C. G.; RETAMAL, J.; MORA, B.; ROA, J. C. \& SÁNCHEZ, R. Frecuencia de virus papiloma humano en tumores no ginecológicos de la Región de La Araucanía, Chile. Int. J. Morphol., 32(4):12541260, 2014.

RESUMEN: El Virus Papiloma Humano (HPV por sus siglas en inglés) es una de las infecciones de transmisión sexual más frecuentes del mundo y se encuentra presente en la mayoría de los cánceres de cuello uterino. Se ha descrito su presencia en otros tipos de cáncer no ginecológicos como lo son esófago y próstata. Sin embargo, las frecuencias de HPV descritas hasta el momento para estos tipos de cáncer son muy variables, y no hay artículos donde se muestren la presencia de HPV en estas neoplasias en Chile. El objetivo de este estudio fue determinar la frecuencia de HPV en muestras de biopsias de tumores no ginecológicos y tejido inflamatorio de pacientes de la región de La Araucanía. Se extrajo DNA desde un total de 47 biopsias de pacientes con esofagitis, 25 con carcinoma escamoso esofágico, 20 con hiperplasia nodular de la próstata y 39 con adenocarcinoma prostático. Estas fueron analizadas por PCR de la región L1 del virus y posterior genotipificación por reverse line blot. Se detectó HPV en el 53,2\% de las muestras de esofagitis, 48\% en muestras de carcinoma escamoso esofágico, $15 \%$ en hiperplasia nodular de la próstata y un 15,4\% en los casos de adenocarcinoma prostático. Siendo los más frecuentes los genotipos de HPV 16 y 18, ya sea en infecciones simples o junto con otros genotipos, en lesiones preneoplásicas y neoplásicas de los tejidos estudiados. Existe una alta frecuencia de infección por HPV en biopsias de esofagitis y tejido inflamatorio esofágico de pacientes de la región de la Araucanía. En los casos de adenocarcinoma prostático e hiperplasia nodular de la próstata se observa una baja frecuencia de HPV.

PALABRAS CLAVE: Virus Papiloma Humano; Carcinoma esofágico de células escamosas; Adenocarcinoma de próstata; Reverse Line Blot.

\section{INTRODUCCIÓN}

El Virus Papiloma Humano (HPV por sus siglas en inglés) es una de las infecciones de transmisión sexual más frecuentes del mundo (Dunne et al., 2006), siendo el principal agente etiológico del cáncer cervicouterino, encontrándose presente en el 99,7\% de los casos de esta neoplasia (Walboomers et al., 1999).

Hasta el momento se han descrito más de 200 tipos virales de HPV, 40 de éstos presentan tropismo por el área anogenital y mucosas, pudiendo ser agrupados como HPV de bajo riesgo y alto riesgo oncogénico, éstos últimos asociados con lesiones de alto grado o carcinomas invasores (Woodman et al., 2007). Los genotipos más importantes de HPV de bajo riesgo oncogénico son: HPV $6,11,40,42,43,44,54,61,72$ y 81 , de los cuales los más frecuentemente encontrados son los tipos 6 y 11 , que producen lesiones benignas denominadas condilomas en hombres y mujeres (Burd, 2003). Dentro de los genotipos

\footnotetext{
* Laboratorio de Patología Molecular, Departamento de Anatomía Patológica, BIOREN-CEGIN, Facultad de Medicina, Universidad de La Frontera, Temuco, Chile.

** Laboratorio de Virus Oncológicos, CEGIN, Facultad de Medicina, Universidad de La Frontera, Temuco Chile.

*** Departamento de Patología, Pontificia Universidad Católica de Chile, Santiago, Chile.

Esta investigación fue financiada por el Proyecto MEC N 80110037, Proyecto CORFO N 12IDL2-18157, Proyecto DIUFRO DI13-0039, Proyecto CORFO-CEGIN N ${ }^{\circ}$ 09CN14-5960, Scientific and Technological Bioresource Nucleus (BIOREN); Proyecto FONDECYT Postdoctoral $\mathrm{N}^{\circ} 3130630$ (C. Ili).
} 
HOFFSTETTER, R.; ANDANA, A.; GUZMÁN, P.; ILI, C. G.; RETAMAL, J.; MORA, B.; ROA, J. C. \& SÁNCHEZ, R. Frecuencia de virus papiloma humano en tumores no ginecológicos de la Región de La Araucanía, Chile. Int. J. Morphol., 32(4):1254-1260, 2014.

de HPV de alto riesgo oncogénico están los tipos 16, 18, $31,33,34,35,39,45,51,52,56,58,59,66,68,70,73$ y 82 , ya que se encuentran presentes en lesiones de alto grado y carcinomas, especialmente los tipos 16 y 18 (Burd).

Además, se ha descrito que este virus está presente en otros tipos de cáncer, principalmente de la región anogenital de hombres y mujeres, tales como: cáncer de vulva, ano, uretra, recto, próstata, entre otros. Por otro lado, también se puede encontrar en pacientes con cáncer de cavidad oral (cáncer de cuello y cabeza, amígdalas) y de las vía digestiva y respiratoria, como es el caso del cáncer de faringe, laringe y esófago (Giuliano et al., 2008; Petersen \& Klein, 2008).

A nivel mundial, el adenocarcinoma de próstata (AP) es la segunda neoplasia con mayor cantidad de casos nuevos y la sexta causa de muerte por cáncer, en varones (Jemal et al., 2011). En Chile, es el cáncer de mayor incidencia y la segunda causa de muerte por neoplasias en hombres, con una mortalidad de 23,6 casos por cada 100.000 hombres (Ferlay et al., 2013). El HPV y AP fueron relacionados por primera vez el año 1990 por McNicol \& Dodd (1991). Diversos estudios describen la presencia de HPV en esta neoplasia, donde se observan rangos de frecuencias de infección por HPV entre 4,2 y 58,3\% de los casos, debido a los diversos tipos de metodología usadas (Al Moustafa, 2008; Leiros et al., 2005).

El carcinoma epidermoide esofágico (CEE) es la octava neoplasia más común y la sexta causa de muerte por cáncer, a nivel mundial (Ferlay et al.; Parkin et al., 2005). Tiene claras diferencias en la incidencia, mortalidad y prevalencia en hombres y mujeres, de acuerdo al área geográfica analizada, siendo considerado Chile (Syrjänen, 2002) y Sudamérica como una zona geográfica de alto riesgo de desarrollar esta enfermedad (Parkin, et al., 2005). Los rangos de detección de HPV en CEE son altamente variables entre diferentes regiones geográficas alrededor del mundo, siendo significativas en regiones con alto riesgo de cáncer de esófago (Syrjänen).

En Chile, hay poca información que analice la presencia de HPV en tumores de esófago y próstata. En el caso del CEE sólo existe un artículo donde se analizaron 26 casos chilenos, encontrándose un $19 \%$ de positividad de DNA de HPV 16 (Castillo et al., 2006). Para el AP no se encontró referencia alguna en Chile. Por ello, en el presente estudio se planteó como objetivo establecer la frecuencia de infección por HPV en tumores no ginecológicos y tejido inflamatorio, tales como los de esófago y próstata.

\section{MATERIAL Y MÉTODO}

Muestras clínicas. Las muestras fueron obtenidas a partir de material de archivo, correspondientes a biopsias (quirúrgicas o endoscópicas) fijadas en formalina e incluidas en parafina, provenientes de la Unidad de Anatomía Patológica y Citopatología del Hospital Doctor Hernán Henríquez Aravena, Temuco, Chile. Un total de 72 tejidos de esófago y 59 de próstata fueron analizados. De estas muestras, 25 correspondían a CEE, 47 fueron de pacientes con esofagitis, 39 correspondían a AP y 20 provenían de pacientes con hiperplasia nodular de la próstata.

Extracción de DNA. Cinco cortes de $10 \mathrm{~mm}$ de las biopsias recolectadas, fueron desparafinados con xilol y diluciones seriadas de etanol absoluto. Posteriormente, se realizó una microdisección manual de la zona de la lesión marcada en una lámina teñida con hematoxilinaeosina (HE) por un médico patólogo (PG). El tejido fue digerido en buffer de lisis con proteinasa $\mathrm{K}(10 \mathrm{mg} / \mathrm{mL})$, para luego realizar la extracción de DNA con columnas utilizando el kit E.Z.N.A.® Tissue DNA (Omega BioTek Ink.). Para comprobar la integridad del DNA obtenido, se amplificó un fragmento de 268 pb del gen de la beta-globina usando los partidores GH20 y PCO4 y las condiciones previamente descritas (Aedo et al., 2007).

Genotipificación. Para la detección de HPV se amplificó el fragmento L1 del genoma viral, mediante PCR anidado usando los partidores MY09 - MY11 y GP5+GP6+. El cebador GP6+ se encontraba biotinilado, de acuerdo a lo descrito anteriormente (van den Brule et al., 2002), para posteriormente proseguir con la genotipificación del virus, mediante la técnica de reverse line blot. Se usaron oligosondas específicas para los genotipos (cerca de 40 genotipos), de acuerdo a lo descrito previamente (Ili et al., 2011; van den Brule, et al.). Se utilizó el sistema de miniblotter MN45 (Inmunetics, Boston, MA, EE.UU) para cargar las muestras. Para la visualización se utilizó el conjugado peroxidasaestreptadivina (Roche, Basel, Suiza), para finalmente revelar mediante quimioluminicescia con ECL (Amersham, Bioscience, Piscataway, NY, EE.UU) en un hiperfilm (GE Health care, Uppsala, Suecia).

Muestras Control. Como control positivo de los HPV $6,11,16$ y 18 se utilizaron clones de plásmidos (ATCC) provistos por el Dr. Peter Snijders (VU University Medical Center, Amsterdam, Holanda). El control negativo consistió en DNA genómico sin HPV. Además se agregaron blancos en los cuales se utilizó agua desionizada en vez de DNA en el mix de PCR. 
Definición de Infecciones de HPV simples o múltiples. Como describió previamente Ili et al., las infecciones únicas fueron definidas como la detección de una sola señal positiva de un genotipo de HPV mientras que las infecciones múltiples correspondieron a la presencia de dos o más señales positivas.

Análisis Estadístico. Se realizó la prueba de Fisher para comparar dos grupos de muestras, con un 95\% de confianza. Para el análisis de las características clínicopatológicas de las muestras se utilizó el test no paramétrico de Mann Whitney, con un $95 \%$ de confianza. Para realizar estos análisis se utilizó el programa Prisma GraphPad versión 5.0 para Windows.

\section{RESULTADOS}

Características clínicopatológicas de las muestras. El genotipificado de HPV fue realizado en 72 biopsias de esófago y 59 biopsias de próstata. La edad promedio de los pacientes al momento de la toma de muestra fue de 58,$9 ; 69,9 ; 70,5$ y 66,8 años para el grupo con esofagitis, CEE, hiperplasia nodular de la próstata y AP, respectivamente (Tabla I). Los pacientes fueron distribuidos en dos grupos de edades: $\leq 60$ años y $>60$ años para las muestras de Esófago y entre $\leq 70$ años y > 70 años para las muestras de próstata. Cuando se compararon los grupos de lesiones y cánceres de cada tejido con respecto a la edad, sólo se observó una diferencia estadísticamente significativa entre las muestras de esofagitis y las de CEE (P value $=0,0023$ ). Sin embargo, no se observó diferencia significativa entre las muestras de hiperplasia nodular de la próstata y AP.

$\mathrm{Al}$ analizar el tamaño de los tumores de próstata de acuerdo a la edad no hay diferencias significativas entre los grupos de pacientes de edades entre menores de 70 y mayores de 70 años. En cuanto a los tumores de esófago no se pudo ver una diferencia significativa, en la localización de éstos en el esófago (próximo, medio o distal), entre grupos de pacientes menores o iguales a 60 y mayores a 60. Se usó la prueba no-paramétrica Mann Withney, con un $95 \%$ de confianza.

Detección de HPV. Todas las muestras tuvieron una buena integridad de DNA usando el ensayo de amplificación de beta-globina. El PCR anidado del fragmento L1, de todas las muestras, fue seguido por la hibridación en reverse line blot. Se encontró DNA de HPV en el 51,4\% (37/72) de las muestras de esófago y un 15,2\% (9/59) de las muestras de próstata. Además, en este análisis se de- tectó HPV en un 53,2\% (25/47) de las muestras de esofagitis y en un $48 \%$ (12/25) de los casos de CEE. En muestras de hiperplasia nodular de la próstata se encontró un $15 \%$ (3/20) de casos positivos para HPV y un $15,4 \%$ (6/39) en los casos de AP. Al comparar la presencia de DNA de HPV en los grupos de edades de la Tabla I, no se encontraron diferencias significativas, descartando así, el efecto de la edad sobre la infección por HPV, en nuestros grupos de muestras.

Genotipificación de HPV en muestras esófago. Se encontró presencia de DNA de HPV en el 53,2\% de las biopsias de esofagitis (Tabla II). Se observó un 30\% (16/ 47) de infecciones únicas y un 19,1\% (9/47) de infecciones múltiples en este tipo de muestra. En el $96 \%$ de los casos positivos para HPV se encontró un genotipo de alto riesgo oncogénico. El genotipo más frecuente en las infecciones simples fue HPV 16 con un 19,1\% (9/47), seguido por los genotipos HPV 18, 51 y 58 con un 4,3\% (2/ 47) y por último HPV 11 con un $2,1 \%$ (1/47). En cuanto a las infecciones múltiples la mayor frecuencia se dio por la presencia de HPV 16 en conjunto con otros genotipos con un 8,5\% (4/47), seguido por HPV 16 en combinación con HPV 18 con un 6,4\% (3/47) y por último la combinación de HPV 18 con otros genotipos y la combinación de HPV 16, 18 y otro con 2,1\% (1/47). En los casos de CEE se encontró presencia de HPV en un 48\% (12/25) de las biopsias. De estas muestras positivas se detectó un $28 \%$ (7/25) de infecciones únicas y un 20\% (5/25) de infecciones múltiples. El $91.7 \%$ de los casos positivos para HPV poseían un genotipo de alto riesgo. Dentro de las infecciones únicas, los genotipos más frecuentes de HPV fueron 18 y 6 con una frecuencia de $24 \%$ (6/25) y $4 \%$ (1/ $25)$, respectivamente. De las infecciones múltiples, la combinación de HPV 16 y 18 con otro tipo viral, y la combinación de HPV 18 con otro genotipo fueron los más frecuentes con un $8 \%(2 / 25)$, la combinación de HPV 16 y 18 tuvo una frecuencia del $4 \%(1 / 25)$.

Genotipificación de HPV en muestras de próstata. Se encontró un $15 \%$ de positividad para DNA de HPV en casos de hiperplasia nodular de la próstata (Tabla III), los cuales correspondieron en su totalidad a infecciones múltiples de HPV, viéndose involucrados los genotipos 16 , 18 en mayor medida con un $10 \%(2 / 20)$ cada uno en diversas combinaciones. En adenocarcinoma prostático el $15,4 \%$ (6/39) de las biopsias presentaron positividad para DNA de HPV, observándose sólo infecciones únicas. Solamente se detectaron los genotipos de HPV 16 y 18 con un $7,7 \%(3 / 39)$ cada uno.

Análisis Estadístico. No se observó ninguna diferencia significativa entre los distintos grupos en estudio. 
HOFFSTETTER, R.; ANDANA, A.; GUZMÁN, P.; ILI, C. G.; RETAMAL, J.; MORA, B.; ROA, J. C. \& SÁNCHEZ, R. Frecuencia de virus papiloma humano en tumores no ginecológicos de la Región de La Araucanía, Chile. Int. J. Morphol., 32(4):1254-1260, 2014.

Tabla I. Distribución de edades en los distintos grupos de pacientes con lesiones o cáncer.

\begin{tabular}{cccccccccc}
\hline & \multicolumn{2}{c}{ Esofagitis } & \multicolumn{2}{c}{$\begin{array}{c}\text { Carcinoma } \\
\text { Esofágico }\end{array}$} & & $\begin{array}{c}\text { Hiperplasia Nodular } \\
\text { de la Próstata }\end{array}$ & \multicolumn{2}{c}{$\begin{array}{c}\text { Adenocarcinoma } \\
\text { Prostático }\end{array}$} \\
\hline Edades & $\mathrm{n}$ & $\%$ & $\mathrm{n}$ & $\%$ & Edades & $\mathrm{n}$ & $\%$ & $\mathrm{n}$ & $\%$ \\
$\leq 60$ años & 23 & 49 & 6 & 24 & $\leq 70$ años & 10 & 50 & 25 & 64 \\
$>60$ años & 24 & 51 & 19 & 76 & $>70$ años & 10 & 50 & 14 & 36 \\
Total & 47 & --- & 25 & --- & Total & 20 & --- & 39 & --- \\
\hline
\end{tabular}

Tabla II. Frecuencia de infección por HPV en biopsias de pacientes con esofagitis y carcinoma esofágico-

\begin{tabular}{|c|c|c|c|c|c|c|}
\hline \multirow[b]{2}{*}{$\begin{array}{l}\text { Tipo de infección por } \\
\text { HPV }\end{array}$} & \multicolumn{3}{|c|}{\begin{tabular}{|c|} 
Esofagitis \\
Porcentaje de HPV
\end{tabular}} & \multicolumn{3}{|c|}{$\begin{array}{l}\text { Cáncer Esofágico } \\
\text { Porcentaje de HPV }\end{array}$} \\
\hline & $\mathrm{n}$ & $\begin{array}{c}\text { Sobre esofagitis o } \\
\text { gastritis }^{\mathrm{A}}\end{array}$ & $\begin{array}{c}\text { Sobre IS o IM de } \\
\text { esofagitis o gastritis }{ }^{\text {B }}\end{array}$ & $\mathrm{n}$ & $\begin{array}{l}\text { Sobre cáncer } \\
\text { esofágico }^{\mathrm{C}}\end{array}$ & $\begin{array}{l}\text { Sobre IS o IM de } \\
\text { cáncer esofá gico }{ }^{\mathrm{D}}\end{array}$ \\
\hline Casos Negativos de HPV & 22 & 46,8 & --- & 13 & 52,0 & -- \\
\hline Infecciones Simples & 16 & 34,0 & 100 & 7 & 28,0 & 100 \\
\hline HPV 16 & 9 & 19,1 & 56,3 & 0 & 0 & -- \\
\hline HPV 18 & 2 & 4,3 & 12,5 & 6 & 24,0 & 85,7 \\
\hline HPV 51 & 2 & 4,3 & 12,5 & 0 & 0 & -- \\
\hline HPV 58 & 2 & 4,3 & 12,5 & 0 & 0 & -- \\
\hline HPV 6 & 0 & 0 & --- & 1 & 4,0 & 14,3 \\
\hline HPV 11 & 1 & 2,1 & 6,3 & 0 & 0 & -- \\
\hline Infecciones Múltiples & 9 & 19,1 & 100 & 5 & 20,0 & 100 \\
\hline HPV $16+18$ & 3 & 6,4 & 33,3 & 1 & 4,0 & 20,0 \\
\hline HPV $16+$ otro & 4 & 8,5 & 44,4 & 0 & 0 & -- \\
\hline HPV 18 + otro & 1 & 2,1 & 11,1 & 2 & 8,0 & 40,0 \\
\hline HPV $16+18+$ otro & 1 & 2,1 & 11,1 & 2 & 8,0 & 40,0 \\
\hline Total & 47 & --- & --- & 25 & --- & -- \\
\hline
\end{tabular}

Tabla III. Frecuencia de infección por HPV en biopsias de pacientes con hiperplasia nodular de la próstata y adenocarcinoma prostático.

\begin{tabular}{|c|c|c|c|c|c|c|}
\hline \multirow[b]{3}{*}{ Tipo de infección por HPV } & \multirow{2}{*}{\multicolumn{3}{|c|}{$\begin{array}{c}\text { Hiperplasia Nodular de la Próstata } \\
\text { Porcentaje de HPV }\end{array}$}} & \multirow{2}{*}{\multicolumn{3}{|c|}{$\begin{array}{c}\text { Adenocarcinoma Prostático } \\
\text { Porcentaje de HPV }\end{array}$}} \\
\hline & & & & & & \\
\hline & $\mathrm{n}$ & $\begin{array}{c}\text { Sobre } \\
\text { prostatitis }\end{array}$ & $\begin{array}{l}\text { Sobre IS o IM de } \\
\text { prostatitis }^{\text {B }}\end{array}$ & $\mathrm{n}$ & $\begin{array}{l}\text { Sobre A. } \\
\text { prostático }\end{array}$ & $\begin{array}{l}\text { Sobre IS o IM de } \\
\text { A. prostático }{ }^{D}\end{array}$ \\
\hline Casos Negativos de HPV & 17 & 85,0 & --- & 33 & 84,6 & --- \\
\hline Infecciones Simples & 0 & 0 & --- & 6 & 15,4 & 100 \\
\hline HPV 16 & 0 & 0 & --- & 3 & 7,7 & 50,0 \\
\hline HPV 18 & 0 & 0 & --- & 3 & 7,7 & 50,0 \\
\hline Infecciones Múltiples & 3 & 15.0 & 100 & 0 & 0 & --- \\
\hline HPV $16+$ otro & 1 & 5,0 & 33,3 & 0 & 0 & --- \\
\hline HPV $18+$ otro & 1 & 5,0 & 33,3 & 0 & 0 & --- \\
\hline HPV $16+18+$ otro & 1 & 5,0 & 33,3 & 0 & 0 & --- \\
\hline Total & 20 & --- & --- & 39 & --- & --- \\
\hline
\end{tabular}

\section{DISCUSIÓN}

El HPV es una de las infecciones de transmisión sexual más comunes alrededor del mundo y ha sido relacionado con diversos tipos de cáncer principalmente ginecológicos. Hay pocos estudios en otros tipos de cáncer no ginecológicos como en los de cabeza y cuello, orofaríngeo, laringe, próstata y esó- fago (Giuliano et al.; Petersen \& Klein), no existiendo en Chile estudios al respecto.

La frecuencia de DNA de HPV en biopsia fue similar a otras regiones con alto riesgo de $\mathrm{CEE}$ y de alto riesgo 
de infección por HPV, con un 48\%, similar al 50\% descrito en la región de Shantou, China (Zhang et al., 2010), y $56,1 \%$ en diferentes regiones de China (Wang et al., 2010). Previamente se había reportado una asociación entre los genotipos de HPV 16 y 18, y el alto riesgo de desarrollar carcinoma esofágico de células escamosas (Zhang et al., 2011), encontrando resultados similares a lo observado previamente en lesiones cervicales (Ili et al.). En el presente estudio también se encontró una alta frecuencia de HPV 16 y 18 , ya que contabilizando su presencia en infecciones únicas y múltiples, éstos se encontraron presentes en el $42,4 \%$ de las muestras de esofagitis, correspondientes al $91 \%$ de las muestras positivas para HPV en ese tipo de muestras, mientras que en carcinoma esofágico se encontraron los genotipos 16 y 18 en el $44 \%$ de las muestras, que corresponde al $91,7 \%$ de los casos positivos en cáncer esofágico.

Si bien la frecuencia de HPV 16 en esofagitis decae al evaluarla en CEE, de un $36 \%$ a un $12 \%$, esto puede deberse al bajo número de muestras de $\mathrm{CEE}$, con respecto a la frecuencia de HPV 18, se ve un aumento de la frecuencia, pasando de un $15 \%$ en esofagitis a un $44 \%$ en CEE, pero como es un bajo número de muestras, solo podría especularse de que el HPV18 este más relacionado con la carcinogénesis esofágica.

La alta frecuencia de DNA de HPV en las biopsias de esofagitis puede ser debido a que es un precursor del CEE, como lo que pasa con las lesiones intraepiteliales del cuello uterino. Syrjänen, en el año 2002, encontró una alta frecuencia de HPV en esofagitis crónica, descrito como el hallazgo más frecuente en poblaciones con alto riesgo de desarrollar CEE, como lo son poblaciones de Irán y China. Existen otros factores asociados a la carcinogénesis de CEE como la región geográfica, el consumo de cigarros, edad, hábitos alimenticios, carga viral, integración del virus HPV e infecciones múltiples (Zhang et al., 2011).

Algunos factores que podrían afectar son la distribución geográfica, el tipo de comida (Castellsagué et al., 2000; Kamangar et al., 2009), cigarro (Kamangar et al.; Parkin, et al.), alcoholismo (Kamangar et al.; Yokoyama \& Omori, 2005), historia familiar previa (Chang-Claude et al., 1997) y el virus papiloma humano también ha sido postulado (Zhang et al., 2010).

Recientemente, se publicó un meta-análisis realizado por Liyanage et al. (2013), donde analizaron los resultados de 21 estudios de caso y control de cáncer de esófago, utilizando como control, sujetos normales sin ningún condición médica preexistente o crónica. Con un total de 1223 casos y 1415 controles, de distintos países, con distintos niveles de incidencia de esta neoplasia. Como resultado principal, se obtuvo una asociación de la infección por HPV y el CEE, además su estudio sugería que el HPV incrementaba tres veces el riesgo de CEE (odd ratio de 3,042, 95\% de confianza, intervalo de confianza de 2,2 a 4,2), sugiriendo así que el HPV pudiera tener un rol etiológico, encontrándose una relación más fuerte en países con una incidencia baja o media como lo son Grecia, Estados Unidos y Australia, comparándolos con reportes provenientes de regiones con alta incidencia de CEE como lo son China, Irán y Sudáfrica.

La presencia de HPV en carcinoma prostático fue descrita por primera vez por McNicoll \& Dodd en el año 1991, encontrándose presente en el 58,3\% (14/27) de las muestras de carcinoma prostático, y en 60,7\% (34/56) de hiperplasias benignas de próstata, no encontrando diferencias significativas entre los grupos. Leiros et al. realizaron estudios en Argentina encontrando un 41,5\% (17/41) de positividad de HPV en muestras de carcinoma prostático y $0 \%$ en hiperplasias de próstata. En nuestro estudio tampoco encontraron diferencias significativas entre los casos de hiperplasia nodular de la próstata y de adenocarcinoma prostático, además de que se encontró una baja positividad para DNA de HPV en comparación a otros estudios, lo cual podría ser debido a otros factores como la región geográfica, la edad y comportamiento sexual, entre otros. Además hay que considerar que los casos controles fueron muestras de hiperplasia nodular de la próstata en la cual, ya existe un proceso inflamatorio de la próstata, por lo cual no es una muestra normal.

En conclusión, no se observó una diferencia significativa entre los grupos pareados de esófago y próstata. Sin embargo se observa una elevada frecuencia de HPV en cáncer de esófago y esofagitis. Mientras que en muestras de próstata si bien de detectó HPV la frecuencia fue baja. Sin embargo, es necesario un estudio más completo con un mayor número de muestras, para determinar si existe relación de esta infección con la carcinogénesis de próstata y esófago, además de una comparación con muestras de individuos normales.

HOFFSTETTER, R.; ANDANA, A.; GUZMÁN, P.; ILI, C. G.; RETAMAL, J.; MORA, B.; ROA, J. C. \& SÁNCHEZ, R. Human papilloma virus frequency in non-gynecological tumors in the Araucanía region, Chile. Int. J. Morphol., 32(4):1254-1260, 2014.

SUMMARY: Human Papilloma Virus (HPV) is the most common sexually transmitted disease in the world and it is present in practically all cervical cancers. Its presence was described in 
other types of non-gynecologic cancer such as esophageal and prostate. However, HPV frequency described for these cancers is highly variable, and there are no articles describing the presence of HPV in these tumors in Chile. To determine HPV frequency in samples from biopsies of non-gynecological tumors and inflammatory tissue from patients in the Araucanía region, DNA was extracted from a total of 47 biopsies from patients with esophagitis, 25 with esophageal squamous cell carcinoma, 20 with prostate nodular hyperplasia and 39 with prostate adenocarcinoma. These were analyzed by PCR of HPV L1 region and subsequent genotyping by reverse line blot. HPV was detected in $53.2 \%$ of esophagitis samples, $48 \%$ in esophageal squamous cell carcinoma, $15 \%$ in prostatitis and $15.4 \%$ in cases of prostatic adenocarcinoma. The most frequent HPV genotypes were 16 and 18 , either single or in combination with other genotype infections, in inflammatory tissue and neoplastic lesions. In patients of the Araucanía region, there is a high rate of HPV infection in biopsies obtained in esophagitis and esophageal inflammatory tissue. In cases of prostatic adenocarcinoma and prostate nodular hyperplasia a low rate of HPV was observed.

KEY WORDS: Human Papillomavirus; Esophageal neoplasm; Prostate neoplasm; Reverse line blot - assay.

\section{REFERENCIAS BIBLIOGRÁFICAS}

Aedo A. S.; Melo A. A.; García, P.; Guzmán G. P.; Capurro V. I. \& Roa S. J. C. Detección y tipificación de virus papilloma humano en lesiones preneoplásicas del cuello uterino mediante PCR-RFLP. Rev. Med. Chile, 135(2):167-73, 2007.

Al Moustafa, A. E. Involvement of human papillomavirus infections in prostate cancer progression. Med. Hypotheses, 71(2):209-11, 2008.

Burd, E. M. Human papillomavirus and cervical cancer. Clin. Microbiol. Rev., 16(1):1-17, 2003.

Castellsagué, X.; Muñoz, N.; De Stefani, E.; Victora, C. G.; Castelletto, R. \& Rolón, P. A. Influence of mate drinking, hot beverages and diet on esophageal cancer risk in South America. Int. J. Cancer, 88(4):658-64, 2000.

Castillo, A.; Aguayo, F.; Koriyama, C.; Shuyama, K.; Akiba, S.; Herrera-Goepfert, R.; Carrascal, E.; Klinge, G.; Sánchez, J. \& Eizuru, Y. Human papillomavirus in lung carcinomas among three Latin American countries. Oncol Rep., 15(4):883-8, 2006.

Chang-Claude, J.; Becher, H.; Blettner, M.; Qiu, S.; Yang, G. \& Wahrendorf, J. Familial aggregation of oesophageal cancer in a high incidence area in China. Int. J. Epidemiol., 26(6):1159-65, 1997.

Dunne, E. F.; Nielson, C. M.; Stone, K. M.; Markowitz, L. E. \& Giuliano, A. R. Prevalence of HPV infection among men: A systematic review of the literature. J. Infect. Dis., 194(8):104457, 2006.

Ferlay, J.; Soerjomataram, I.; Ervik, M.; Dikshit, R.; Eser, S.; Mathers, C.; Rebelo, M.; Parkin, D. M.; Forman, D. \& Bray, F. GLOBOCAN 2012 v1.0, Cancer Incidence and Mortality Worldwide: IARC CancerBase No. 11 [Internet]. Lyon, International Agency for Research on Cancer, 2013. Disponible en: http://globocan.iarc.fr

Giuliano, A. R.; Lazcano-Ponce, E.; Villa, L. L.; Flores, R.; Salmeron, J.; Lee, J. H.; Papenfuss, M. R.; Abrahamsen, M.; Jolles, E.; Nielson, C. M.; Baggio, M. L.; Silva, R. \& Quiterio, M. The human papillomavirus infection in men study: human papillomavirus prevalence and type distribution among men residing in Brazil, Mexico, and the United States. Cancer Epidemiol. Biomarkers Prev., 17(8):2036-43, 2008.

Ili, C. G.; Brebi, P.; López, J.; García, P.; Leal, P.; Suarez, E. \& Roa, J. C. Genotyping of human papillomavirus in cervical intraepithelial neoplasia in a high-risk population. J. Med. Virol., 83(5):833-7, 2011.

Jemal, A.; Bray, F.; Center, M. M.; Ferlay, J.; Ward, E. \& Forman, D. Global cancer statistics. CA Cancer J. Clin., 61(2):69-90, 2011.

Kamangar, F.; Chow, W. H.; Abnet, C. C. \& Dawsey, S. M. Environmental causes of esophageal cancer. Gastroenterol. Clin. North Am., 38(1):27-57, 2009.

Leiros, G. J.; Galliano, S. R.; Sember, M. E.; Kahn, T.; Schwarz, E. \& Eiguchi, K. Detection of human papillomavirus DNA and p53 codon 72 polymorphism in prostate carcinomas of patients from Argentina. BMC Urol., 5:15, 2005.

Liyanage, S. S.; Rahman, B.; Ridda, I.; Newall, A. T.; Tabrizi, S. N.; Garland, S. M.; Segelov, E.; Seale, H.; Crowe, P. J.; Moa, A. \& Macintyre, C. R. The aetiological role of human papillomavirus in oesophageal squamous cell carcinoma: a meta-analysis. PLoS One, 8(7):e69238, 2013.

McNicol, P. J. \& Dodd, J. G. High prevalence of human papillomavirus in prostate tissues. J. Urol., 145(4):850-3, 1991.

Parkin, D. M.; Bray, F.; Ferlay, J. \& Pisani, P. Global cancer statistics, 2002. CA Cancer J. Clin., 55(2):74-108, 2005.

Petersen, I. \& Klein, F. HPV in non-gynecological tumors. Pathologe, 29( Suppl. 2):118-22, 2008.

Syrjänen, K. J. HPV infections and oesophageal cancer. J. Clin. Pathol., 55(10):721-8, 2002.

van den Brule, A. J.; Pol, R.; Fransen-Daalmeijer, N.; Schouls, L. M.; Meijer, C. J. \& Snijders, P. J. GP5+/6+ PCR followed by reverse line blot analysis enables rapid and high-throughput identification of human papillomavirus genotypes. J. Clin. Microbiol., 40(3):779-87, 2002. 
HOFFSTETTER, R.; ANDANA, A.; GUZMÁN, P.; ILI, C. G.; RETAMAL, J.; MORA, B.; ROA, J. C. \& SÁNCHEZ, R. Frecuencia de virus papiloma humano en tumores no ginecológicos de la Región de La Araucanía, Chile. Int. J. Morphol., 32(4):1254-1260, 2014.

Walboomers, J. M.; Jacobs, M. V.; Manos, M. M.; Bosch, F. X.; Kummer, J. A.; Shah, K. V.; Snijders, P. J.; Peto, J.; Meijer, C. J. \& Muñoz, N. Human papillomavirus is a necessary cause of invasive cervical cancer worldwide. J. Pathol., 189(1):12-9, 1999.

Wang, X.; Tian, X.; Liu, F.; Zhao, Y.; Sun, M.; Chen, D.; Lu, C.; Wang, Z.; Shi, X.; Zhang, Q.; Zhang, D.; Shen, Z.; Li, F.; Harris, C. C.; Cai, H. \& Ke, Y. Detection of HPV DNA in esophageal cancer specimens from different regions and ethnic groups: a descriptive study. BMC Cancer, 10:19, 2010.

Woodman, C. B.; Collins, S. I. \& Young, L. S. The natural history of cervical HPV infection: unresolved issues. Nat. Rev. Cancer, 7(1):11-22, 2007.

Yokoyama, A. \& Omori, T. Genetic polymorphisms of alcohol and aldehyde dehydrogenases and risk for esophageal and head and neck cancers. Alcohol, 35(3):175-85, 2005.

Zhang, D.; Zhang, Q.; Zhou, L.; Huo, L.; Zhang, Y.; Shen, Z. \& Zhu, Y. Comparison of prevalence, viral load, physical status and expression of human papillomavirus-16, -18 and -58 in esophageal and cervical cancer: a case-control study. $B M C$ Cancer, 10:650, 2010.

Zhang, Q. Y.; Zhang, D. H.; Shen, Z. Y.; Xu, L. Y.; Li, E. M. \& Au, W. W. Infection and integration of human papillomavirus in esophageal carcinoma. Int. J. Hyg. Environ. Health, 214(2):156-61, 2011.

\author{
Dirección para Correspondencia: \\ Dr. Raúl Sánchez \\ Laboratorio de Virus Oncológicos, CEGIN \\ Facultad de Medicina \\ Universidad de La Frontera \\ Av. Alemania 0458 Tercer Piso \\ Temuco, \\ CHILE
}

Email: raul.sanchez@ufrontera.cl

Recibido : 09-07-2014

Aceptado: 23-09-2014 